\title{
Study on convergence and stability of a conservative difference scheme for the generalized Rosenau-KdV equation
}

\author{
Jun Zhou ${ }^{\mathrm{a}, *}$, Maobo Zheng ${ }^{\mathrm{b}}$, Xiaomin Daic \\ a School of Mathematics and Statistics, Yangtze Normal University, Chongqing 408100, China. \\ ${ }^{b}$ Chengdu Technological University, Chengdu 610031, China. \\ ${ }^{c}$ Mathematics Teaching and Research Group, Fifth Middle school of Fuling, Chongqing 408000, China.
}

Communicated by X.-J. Yang

\begin{abstract}
In this paper, a conservative nonlinear implicit finite difference scheme for the generalized Rosenau-KdV equation is studied. Convergence and stability of the proposed scheme are proved by a discrete energy method. The proof with a priori error estimate shows that the convergence rates of numerical solutions are both the second order on time and in space. Meanwhile, numerical experiments are carried out to verify the theoretical analysis and show that the scheme is efficient and reliable. (C)2017 All rights reserved.
\end{abstract}

Keywords: Rosenau-KdV equation, finite difference scheme, conservation, convergence, stability. 2010 MSC: 65M06, 65N30.

\section{Introduction}

In this paper, we consider the following generalized Rosenau-KdV equation,

$$
u_{t}+u_{x}+u_{x x x}+u_{x x x t}+\left(u^{p}\right)_{x}=0
$$

where $p \geqslant 2$ is an integer. When $p=2$, Eq. (1.1) is called Rosenau-KdV equation as usual.

To address mathematical or physical aspects of nonlinear models, various analytical methods are often proposed, such as the integral transforms [11,12] and the traveling-wave method [13, 14]. By the usual solitary ansatze method, authors discussed the solitary solutions and gave two invariants for the generalized Rosenau-KdV equation in [4,9]. Two types of soliton solution, whose are, the solitary wave solution and the singular soliton were investigated in [9]. Furthermore, with the help of the perturbation theory and the semi-variation principle, the perturbed generalized Rosenau-KdV equation was discussed analytically. The ansatze method was employed to obtain the topological solution and the shock solution of this equation in [10]. Especially, more solitary solutions of the equation (1.1) were derived by the ansatze method, the $\mathrm{G}^{\prime} / \mathrm{G}$-expansion method as well as the Exp-function method in [3].

\footnotetext{
*Corresponding author

Email addresses: flzjzklm@126.com (Jun Zhou ), 377178554@qq.com (Maobo Zheng), 15823657767@163.com (Xiaomin Dai)
} doi:10.22436/jnsa.010.05.40 
As far as numerical methods are concerned, many numerical techniques are used for the approximation solution of the Rosenau-type equations in $[2,6,8]$, the $\mathrm{KdV}$-type equations and their extensions in $[1,5,15,19]$. Certainly, initial-boundary value conditions must be imposed. In the following, we assume that the boundary condition of the generalized Rosenau-KdV equation (1.1) satisfies

$$
u\left(X_{l}, t\right)=u\left(X_{r}, t\right), u_{x}\left(X_{l}, t\right)=u_{x}\left(X_{r}, t\right)=0, u_{x x}\left(X_{l}, t\right)=u_{x x}\left(X_{r}, t\right)=0, t \in[0, T],
$$

and the initial condition is

$$
u(x, 0)=u_{0}(x) .
$$

Obviously, the assumptions for conditions are in accordance with the Cauchy problem of equation (1.1). In $[7,16]$, two conservative difference schemes for the generalized Rosenau-KdV equation were proposed, while both only discussed one conservative law. Another conservative Crank-Nicolson implicit difference scheme was presented in [18], but the shortcoming exists in the computation for the initial condition, which needs the help of other scheme, such as the average linear scheme (see, for example, [16]). In this paper, we study a new implicit finite difference scheme for the generalized Rosenau-Kdv equation. The corresponding convergence and stability for the scheme are proved by a discrete energy method. With a priori error estimate, the convergence rate $\mathrm{O}\left(\tau^{2}+h^{2}\right)$ of numerical solution is shown.

The rest of this paper is organized as follows. In Section 2, we propose an implicit finite difference scheme for the generalized Rosenau-KdV equation. The convergence and the stability are proved in Section 3. Some numerical tests are given in Section 4 to verify our theoretical analysis. Finally, conclusions are drawn in Section 5.

\section{Conservative implicit difference scheme}

We first give some notations which will be used in next sections and propose the conservative difference scheme for problem (1.1)-(1.3).

As usual, denote $x_{j}=X_{l}+j h, t_{n}=n \tau, 0 \leqslant j \leqslant J, 0 \leqslant n \leqslant N$, where $h=\left(X_{r}-X_{l}\right) / J$ and $\tau$ are the uniform spatial and temporal step size, respectively. Let $u_{j}^{n} \approx u(j h, n \tau), Z_{h}^{0}=\left\{u=\left(u_{j}\right) \mid u_{-1}=u_{0}=u_{J}=\right.$ $\left.\mathfrak{u}_{\mathrm{J}+1}=0,-1 \leqslant j \leqslant J+1\right\}$. Throughout this paper, we denote $C$ as a general constant independent of $h$ and $\tau$. Define difference operators, the inner product, and norms as follows:

$$
\begin{array}{rlrl}
\left(u_{j}^{n}\right)_{x} & =\frac{u_{j+1}^{n}-u_{j}^{n}}{h}, & \left(u_{j}^{n}\right)_{\bar{x}}=\frac{u_{j}^{n}-u_{j-1}^{n}}{h}, & \left(u_{j}^{n}\right)_{\hat{x}}=\frac{u_{j+1}^{n}-u_{j-1}^{n},}{2 h}, \\
\left(u_{j}^{n}\right)_{t}=\frac{u_{j}^{n+1}-u_{j}^{n}}{\tau}, & \left(u_{j}^{n}\right)_{\hat{t}}=\frac{u_{j}^{n+1}-u_{j}^{n-1}}{2 \tau}, & \left(u_{j}^{n}\right)_{x \bar{x}}=\frac{u_{j+1}^{n}-2 u_{j}^{n}+u_{j-1}^{n},}{h^{2}} \\
u_{j}^{n+\frac{1}{2}}=\frac{u_{j}^{n+1}+u_{j}^{n}}{2}, & \left\langle u^{n}, v^{n}\right\rangle=h \sum_{j=1}^{J-1} u_{j}^{n} v_{j}^{n}, & \left\|u^{n}\right\|^{2}=\left\langle u^{n}, u^{n}\right\rangle,\left\|u^{n}\right\|_{\infty}=\max _{0 \leqslant j \leqslant J-1}\left|u_{j}^{n}\right| .
\end{array}
$$

Since $\left(u^{p}\right)_{x}=\frac{2}{1+p} \sum_{i=0}^{p-1} u^{i}\left(u^{p-i}\right)_{x}$ (see, for details, [10]), we can construct the following conservative implicit finite difference scheme for problem (1.1)-(1.3) as follows:

$$
\begin{gathered}
\left(u_{j}^{n}\right)_{t}+\left(u_{j}^{n+1 / 2}\right)_{\hat{x}}+\left(u_{j}^{n+1 / 2}\right)_{x \bar{x} \hat{x}}+\left(u_{j}^{n}\right)_{x x \bar{x} \bar{x} t}+\frac{2}{1+p} \sum_{i=0}^{p-1}\left(u_{j}^{n+1 / 2}\right)^{i}\left[\left(u_{j}^{n+1 / 2}\right)^{p-i}\right]_{\hat{x}}=0, \\
u_{j}^{0}=u_{0}\left(x_{j}\right), 1 \leqslant j \leqslant J-1, \\
u_{0}^{n}=u_{J}^{n}=0, \quad\left(u_{0}^{n}\right)_{\hat{x}}=\left(u_{J}^{n}\right)_{\hat{x}}=0, \quad\left(u_{0}^{n}\right)_{x \bar{x}}=\left(u_{J}^{n}\right)_{\bar{x} x}=0 .
\end{gathered}
$$

\section{Convergence and stability of the scheme}

Firstly, we introduce the discrete Gronwall inequality, the discrete Sobolev inequality, and the discrete summation by parts formula (see [17]). 
Lemma 3.1 (Discrete Gronwall inequality). Suppose $w(k), \rho(k)$ are nonnegative mesh functions and $\rho(k)$ is non-decreasing. If $\mathrm{C}>0$ and $w(\mathrm{k}) \leqslant \rho(\mathrm{k})+\mathrm{C} \tau \sum_{\mathrm{l}=0}^{\mathrm{k}-1} w(\mathrm{l}), \forall \mathrm{k}$, then we have

$$
w(k) \leqslant \rho(k) e^{C \tau k}, \forall k .
$$

Lemma 3.2 (Discrete Sobolev inequality). There exist two constants $C_{1}$ and $C_{2}$ such that

$$
\left\|u^{n}\right\|_{\infty} \leqslant C_{1}\left\|u^{n}\right\|+C_{2}\left\|u_{x}^{n}\right\|
$$

Lemma 3.3. For any two mesh functions $u, v \in Z_{h}^{0}$, one can get,

$$
\left\langle v_{x}, u\right)=-\left\langle v, u_{\bar{x}}\right\rangle,\left\langle u_{\hat{x}}, v\right\rangle=-\left\langle u, v_{\hat{x}}\right\rangle,\left\langle u, v_{x}\right\rangle=-\left\langle u_{x}, v_{x}\right\rangle .
$$

Then we have

$$
\left\langle u, v_{x}\right\rangle=-\left\langle u_{x}, u_{x}\right\rangle=-\left\|u_{x}\right\|^{2} .
$$

Furthermore, if $\left(u_{0}^{n}\right)_{x \bar{x}}=\left(u_{J}^{n}\right)_{x \bar{x}}=0$, then

$$
\left\langle u, u_{x x \bar{x} \bar{x}}\right\rangle=\left\|u_{x x}\right\|^{2} .
$$

Next, we discuss the convergence of scheme (2.1)-(2.3). Let $v_{j}^{n}=v\left(x_{j}, t_{n}\right)$ be the analytical solution of problem (1.1)-(1.3). Then, the truncation error of scheme (2.1)-(2.3) is written as:

$$
r_{j}^{n}=\left(v_{j}^{n}\right)_{t}+\left(v_{j}^{n+\frac{1}{2}}\right)_{\hat{x}}+\left(v_{j}^{n+\frac{1}{2}}\right)_{x \bar{x} \hat{x}}+\left(v_{j}^{n}\right)_{x x \bar{x} \bar{x} t}+\frac{2}{1+p} \sum_{i=0}^{p-1}\left\{\left(v_{j}^{n+1 / 2}\right)^{i}\left[\left(v_{j}^{n+1 / 2}\right)^{p-i}\right]_{\hat{x}}\right\} .
$$

Using the Taylor expansion, it follows that $r_{j}^{n}=\mathrm{O}\left(\tau^{2}+h^{2}\right)$ holds if $\tau, h \rightarrow 0$.

Theorem 3.4. Suppose that $\mathrm{u}_{0} \in \mathrm{H}_{0}^{2}\left[\mathrm{X}_{\mathrm{l}}, \mathrm{X}_{\mathrm{r}}\right], \mathrm{u}(\mathrm{x}, \mathrm{t}) \in \mathrm{C}^{5,3}\left[\mathrm{X}_{\mathrm{l}}, \mathrm{X}_{\mathrm{r}}\right]$. Then the solution $\mathrm{u}^{\mathrm{n}}$ of scheme (2.1)-(2.3) converges to the solution of problem (1.1)-(1.3) and the convergence rate is $\mathrm{O}\left(\tau^{2}+\mathrm{h}^{2}\right)$ by the norm $\|\cdot\|_{\infty}$.

Proof. Subtracting (2.1) from (3.4) and letting $e_{j}^{n}=v_{j}^{n}-u_{j}^{n}$, we have

$$
\begin{aligned}
r_{j}^{n}= & \left(e_{j}^{n}\right)_{t}+\left(e_{j}^{n+\frac{1}{2}}\right)_{\hat{x}}+\left(e_{j}^{n+\frac{1}{2}}\right)_{x \bar{x} \hat{x}}+\left(e_{j}^{n}\right)_{x x \bar{x} \bar{x} t}+\frac{2}{1+p} \sum_{i=0}^{p-1}\left\{\left(v_{j}^{n+\frac{1}{2}}\right)^{i}\left[\left(v_{j}^{n+\frac{1}{2}}\right)^{p-i}\right]_{\hat{x}}\right\} \\
& -\frac{2}{1+p} \sum_{i=0}^{p-1}\left\{\left(u_{j}^{n+\frac{1}{2}}\right)^{i}\left[\left(u_{j}^{n+\frac{1}{2}}\right)^{p-i}\right]_{\hat{x}}\right\} .
\end{aligned}
$$

Taking the inner product of (3.5) with $2 e^{n+\frac{1}{2}}$, that is, $\left(e^{n+1}+e^{\mathfrak{n}}\right)$, we have

$$
\begin{aligned}
\left\langle r^{n}, 2 e^{n+\frac{1}{2}}\right\rangle= & \sum_{j=1}^{J-1}\left\{\left(e_{j}^{n}\right)_{t} \cdot 2 e_{j}^{n+\frac{1}{2}}+\left(e_{j}^{n+\frac{1}{2}}\right)\right\}_{\hat{x}} \cdot 2 e_{j}^{n+\frac{1}{2}}+\left(e_{j}^{n+\frac{1}{2}}\right)_{x x \hat{x}} \cdot 2 e_{j}^{n+\frac{1}{2}} \\
& +\frac{1}{\tau}\left(\left(\left(e_{j}^{n+1}\right)_{x x \bar{x} \bar{x}}\right)-\left(\left(e_{j}^{n}\right)_{x x \bar{x} \bar{x}}\right) \cdot 2 e_{j}^{n+\frac{1}{2}}+\frac{2}{1+p} \sum_{i=0}^{p-1}\left\{\left(v_{j}^{n+1 / 2}\right)^{i}\left[\left(v_{j}^{n+1 / 2}\right)^{p-i}\right]_{\hat{x}} \cdot 2 e_{j}^{n+\frac{1}{2}}\right\}\right. \\
& -\frac{2}{1+p} \sum_{i=0}^{p-1}\left\{\left(v_{j}^{n+1 / 2}\right)^{i}\left[\left(v_{j}^{n+1 / 2}\right)^{p-i}\right]_{\hat{x}} \cdot 2 e_{j}^{n+\frac{1}{2}}\right\} .
\end{aligned}
$$

By the definition of $\left(e_{j}^{n}\right)_{t}$, it follows from the first term of (3.6) on the right side that

$$
\sum_{j=1}^{J-1}\left(\frac{1}{\tau}\left(e_{j}^{n+1}-e_{j}^{n}\right) \cdot 2 e_{j}^{n+\frac{1}{2}}\right)=\frac{1}{\tau}\left(\left\|e^{n+1}\right\|^{2}-\left\|e^{n}\right\|^{2}\right) .
$$


Similarly, with the definition of $e_{\hat{x}}$ and (3.2), for the second and the third term of (3.6), we get

$$
\begin{aligned}
\sum_{j=1}^{J-1}\left(\left(e_{j}^{n+\frac{1}{2}}\right)_{\hat{x}} \cdot 2 e_{j}^{n+\frac{1}{2}}\right) & =\sum_{j=1}^{J-1}\left(\left(e_{j}^{n+\frac{1}{2}}\right)_{x}+\left(e_{j}^{n+\frac{1}{2}}\right)_{\bar{x}}\right) \cdot e_{j}^{n+\frac{1}{2}} \\
& =\sum_{j=1}^{J-1}\left(e_{j}^{n+\frac{1}{2}}\right)_{x} \cdot e_{j}^{n+\frac{1}{2}}+\sum_{j=1}^{J-1}\left(e_{j}^{n+\frac{1}{2}}\right)_{\bar{x}} \cdot e_{j}^{n+\frac{1}{2}}=0,
\end{aligned}
$$

and

$$
\sum_{j=1}^{J-1}\left(e_{j}^{n+\frac{1}{2}}\right)_{x \bar{x} \hat{x}} \cdot 2 e_{j}^{n+\frac{1}{2}}=0 .
$$

According to the boundary conditions (2.3) and (3.3), it follows from the fourth term of (3.6) that

$$
\sum_{j=1}^{J-1} \frac{1}{\tau}\left(\left(e_{j}^{n+1}\right)_{\chi x \bar{x} \bar{x}}-\left(e_{j}^{n}\right)_{\chi x \bar{x} \bar{x}} \cdot 2 e_{j}^{n+\frac{1}{2}}\right)=\frac{1}{\tau}\left(\left\|e_{x x}^{n+1}\right\|^{2}-\left\|e_{x x}^{n}\right\|^{2}\right) .
$$

From (3.6)-(3.10), we get

$$
\left\langle\mathrm{r}^{\mathrm{n}}, 2 e^{\mathrm{n}+\frac{1}{2}}\right\rangle=\frac{1}{\tau}\left(\left\|e^{\mathrm{n}+1}\right\|^{2}-\left\|e^{\mathrm{n}}\right\|^{2}\right)+\frac{1}{\tau}\left(\left\|e_{x x}^{n+1}\right\|^{2}-\left\|e_{x x}^{n}\right\|^{2}\right)+\left\langle\mathrm{Q}_{1}+\mathrm{Q}_{2}, 2 e^{\mathrm{n}+\frac{1}{2}}\right\rangle,
$$

where

$$
\left\{\begin{array}{l}
\mathrm{Q}_{1}=\frac{2}{1+p} \sum_{j=1}^{J} \sum_{i=0}^{p-1}\left\{\left[\left(v_{j}^{n+1 / 2}\right)^{i}-\left(u_{j}^{n+1 / 2}\right)^{i}\right]\left[\left(v_{j}^{n+1 / 2}\right)^{p-i}\right]_{\hat{x}}\right\}, \\
Q_{2}=-\frac{2}{1+p} \sum_{j=0}^{J-1} \sum_{i=0}^{p-1}\left\{\left(u_{j}^{n+1 / 2}\right)^{i}\left[\left(u_{j}^{n+1 / 2}\right)^{p-i}-\left(v_{j}^{n+1 / 2}\right)^{p-i}\right]_{\hat{x}}\right\} .
\end{array}\right.
$$

Therefore, we get

$$
\left(\left\|e^{n+1}\right\|^{2}-\left\|e^{n}\right\|^{2}\right)+\left(\left\|e_{x x}^{n+1}\right\|^{2}-\left\|e_{x x}^{n}\right\|^{2}\right)=\tau\left\langle r^{n}, 2 e^{n+\frac{1}{2}}\right\rangle-\tau\left\langle Q_{1}+Q_{2}, 2 e^{n+\frac{1}{2}}\right\rangle .
$$

According to Theorem 9 in reference [7], Theorem 2.7 in reference [18], and Cauchy-Schwartz inequality, we have

$$
\begin{aligned}
& -\left\langle\mathrm{Q}_{1}, e^{\mathrm{n}+1 / 2}\right\rangle \\
& =\frac{-2}{1+p} \sum_{j=1}^{J}\left\{\sum_{i=0}^{p-1}\left[\left(v_{j}^{n+1 / 2}\right)^{i}-\left(u_{j}^{n+1 / 2}\right)^{i}\right]\left[\left(v_{j}^{n+1 / 2}\right)^{p-i}\right]\right\} e_{j}^{n+1 / 2} \\
& =\frac{-2}{1+p} \sum_{j=1}^{J-1}\left\{\sum_{i=0}^{p-1} e_{j}^{n+1 / 2} \sum_{k=0}^{k-1}\left(v_{j}^{n+1 / 2}\right)^{i-k-1}\left(u_{j}^{n+1 / 2}\right)^{k}\left[\left(v_{j}^{n+1 / 2}\right)^{p-i}\right]\right\} e_{j}^{n+1 / 2} \\
& =\frac{-2}{1+p} \sum_{j=1}^{J-1}\left\{\sum_{i=0}^{p-1}\left[\sum_{k=0}^{k-1} e_{j}^{n+1 / 2}\left(v_{j}^{n+1 / 2}\right)^{i-k-1}\left(u_{j}^{n+1 / 2}\right)^{k}\right]\left[\sum_{k=0}^{p-i-1}\left(v_{j+1}^{n+1 / 2}\right)^{p-i-k-1}\left(v_{j-1}^{n+1 / 2}\right)^{k}\right]\right\} e_{j}^{n+1 / 2} \\
& \leqslant C\left(\left\|e^{n+1}\right\|^{2}+\left\|e^{n+1}\right\|^{2}\right) \\
& \leqslant C\left(\left\|e_{x}^{n+1}\right\|^{2}+\left\|e_{x}^{n}\right\|^{2}+e^{n+1}\left\|^{2}+\right\| e^{n} \|^{2}\right) .
\end{aligned}
$$

Similarly,

$$
-\left\langle Q_{2}, e^{n+1 / 2}\right\rangle \leqslant C\left(\left\|e_{x}^{n+1}\right\|^{2}+\left\|e_{x}^{n}\right\|^{2}+\left\|e^{n+1}\right\|^{2}+\left\|e^{n}\right\|^{2}\right) .
$$


Note that

$$
\left\langle\mathrm{r}^{\mathrm{n}}, 2 \mathrm{e}^{\mathrm{n}+\frac{1}{2}}\right\rangle=\left\langle\mathrm{r}^{\mathrm{n}}, \mathrm{e}^{\mathrm{n}+1}+\mathrm{e}^{\mathrm{n}}\right\rangle \leqslant\left\|\mathrm{r}^{\mathfrak{n}}\right\|^{2}+\frac{1}{2}\left[\left\|e^{\mathrm{n}+1}\right\|^{2}+\left\|e^{\mathfrak{n}}\right\|^{2}\right]
$$

Substituting (3.12)-(3.14) into (3.11), we have

$$
\left(\left\|e^{n+1}\right\|^{2}-\left\|e^{n}\right\|^{2}\right)+\left(\left\|e_{x x}^{n+1}\right\|^{2}-\left\|e_{x x}^{n}\right\|^{2}\right) \leqslant C \tau\left[\left\|e_{x}^{n+1}\right\|^{2}+\left\|e_{x}^{n}\right\|^{2}+\left\|e^{n+1}\right\|^{2}+\left\|e^{n}\right\|^{2}\right]+\tau\left\|r^{n}\right\|^{2} .
$$

Letting $D^{n}=\left\|e^{\mathfrak{n}}\right\|^{2}+\left\|e_{x x}^{n}\right\|^{2}$, from (3.15), we obtain

$$
(1-C \tau)\left(D^{n+1}-D^{n}\right) \leqslant 2 C \tau D^{n}+\tau\left\|r^{n}\right\|^{2} .
$$

If $\tau$ is sufficiently small which satisfies $1-C \tau>0$, then we obtain

$$
\mathrm{D}^{\mathrm{n}+1}-\mathrm{D}^{\mathrm{n}} \leqslant \mathrm{C} \tau \mathrm{D}^{\mathrm{n}}+\mathrm{C} \tau\left\|\mathrm{r}^{\mathrm{n}}\right\|^{2} \text {. }
$$

Summing up in (3.16) from 0 to $n-1$, we get

$$
\mathrm{D}^{\mathrm{n}} \leqslant \mathrm{D}^{0}+\mathrm{C} \tau \sum_{\mathrm{l}=0}^{\mathrm{n}-1} \mathrm{D}^{\mathrm{n}}+\mathrm{C} \tau \sum_{\mathrm{l}=0}^{\mathrm{n}-1}\left\|\mathrm{r}^{\mathrm{l}}\right\|^{2} .
$$

Noticing

$$
\tau \sum_{l=0}^{n-1}\left\|r^{l}\right\|^{2} \leqslant n \tau \max _{0 \leqslant l \leqslant n-l}\left\|r^{l}\right\|^{2} \leqslant T \cdot O\left(\tau^{2}+h^{2}\right)^{2},
$$

from discrete initial conditions, we have $e^{0}=0$ such that $\mathrm{D}^{0}=\mathrm{O}\left(\tau^{2}+\mathrm{h}^{2}\right)^{2}$. Therefore

$$
\mathrm{D}^{\mathrm{n}} \leqslant \mathrm{O}\left(\tau^{2}+\mathrm{h}^{2}\right)^{2}+\mathrm{C} \tau \sum_{\mathrm{l}=0}^{\mathrm{n}-1} \mathrm{D}^{\mathrm{l}}
$$

According to Lemma 3.1, we get $\mathrm{D}^{\mathrm{n}} \leqslant \mathrm{O}\left(\tau^{2}+\mathrm{h}^{2}\right)^{2}$, which implies that

$$
\left\|e^{n}\right\| \leqslant O\left(\tau^{2}+h^{2}\right), \quad\left\|e_{x x}^{n}\right\| \leqslant O\left(\tau^{2}+h^{2}\right) .
$$

It follows from (3.1) that

$$
\left\|e_{x}^{n}\right\| \leqslant O\left(\tau^{2}+h^{2}\right)
$$

By Lemma 3.1, we have

$$
\left\|e^{n}\right\|_{\infty} \leqslant \mathrm{O}\left(\tau^{2}+h^{2}\right) .
$$

This completes the proof of Theorem 3.4.

To prove the stability of the difference scheme, we consider the following initial boundary problem as

$$
\begin{gathered}
u_{t}+u_{x}+u_{x x x}+u_{x x x t}+\left(u^{p}\right)_{x}=w(x, t), \\
u\left(X_{l}, t\right)=u\left(X_{r}, t\right)=0, u_{x}\left(X_{l}, t\right)=u_{x}\left(X_{r}, t\right)=0, u_{x x}\left(X_{l}, t\right)=u_{x x}\left(X_{r}, t\right)=0, t \in[0, T], \\
u(x, 0)=u_{0}(x)+\psi(x), x \in\left[X_{l}, X_{r}\right],
\end{gathered}
$$

where $\omega(x, t)$ and $\psi(x)$ are smooth enough.

We also propose the difference scheme of problem (3.17)-(3.19) given as:

$$
\begin{gathered}
\left(u_{j}^{n}\right)_{t}+\left(u_{j}^{n+1 / 2}\right)_{\hat{x}}+\left(u_{j}^{n+1 / 2}\right)_{x \bar{x} \hat{x}}+\left(u_{j}^{n}\right)_{x x \bar{x} \bar{t}}+\frac{2}{1+p} \sum_{i=0}^{p-1}\left(u_{j}^{n+1 / 2}\right)^{i}\left[\left(u_{j}^{n+1 / 2}\right)^{p-i}\right]_{\hat{x}}+\left(\omega_{j}^{n}\right)_{t}=0, \\
u_{j}^{0}=u_{0}\left(x_{j}\right)+\psi_{j}, \quad 0 \leqslant j \leqslant J-1, \\
u_{0}^{n}=u_{J}^{n}=0,\left(u_{0}^{n}\right)_{\hat{x}}=\left(u_{j}^{n}\right)_{\hat{x}}=0,\left(u_{0}^{n}\right)_{x \bar{x}}=\left(u_{j}^{n}\right)_{\bar{x} x}=0,
\end{gathered}
$$

where $\omega_{j}^{n}=\omega\left(x_{j}, t_{n}\right), \psi_{j}=\psi\left(x_{j}\right)$.

The proof is similar to that of Theorem 3.4. We omit the details and present the stability theorem as follows. 
Theorem 3.5. Suppose that $\left\{u_{j}^{n}\right\}$ is the solution of scheme (2.1)-(2.3) and $\left\{u_{j}^{n}\right\}$ is the solution of scheme (3.20)(3.22). If the mesh step $\mathrm{h}$ and $\tau$ are small enough for $\varepsilon_{j}^{n}=\mathrm{u}_{j}^{n}-\mathrm{u}_{j}^{n}$, then we can get

$$
\left\|\varepsilon^{n}\right\|+\left\|\varepsilon_{x x}^{n}\right\| \leqslant C\left(\|\psi\|^{2}+\tau \sum_{l=0}^{n-1}\left\|\omega^{l}\right\|^{2}\right)
$$

\section{Numerical experiments}

In this section, we present some numerical experiments to verify theoretical analysis obtained in the previous section.

Take $X_{l}=-60$ and $X_{r}=90$, and consider two cases: $p=3$ and $p=5$, respectively.

According to the references $[4,9]$, the soliton solution with $p=3$ is as follows:

$$
u(x, t)=\frac{1}{4} \sqrt{-15+3 \sqrt{41}} \operatorname{sech}^{2} \frac{1}{4} \sqrt{\frac{-5+\sqrt{41}}{2}}\left[x-\frac{1}{10}(5+\sqrt{41}) t\right]
$$

with the given initial condition

$$
u(x, 0)=\frac{1}{4} \sqrt{-15+3 \sqrt{41}} \operatorname{sech}^{2} \frac{1}{4} \sqrt{\frac{-5+\sqrt{41}}{2}} x .
$$

For $p=5$, we have the soliton solution,

$$
u(x, t)=\sqrt[4]{\frac{4}{15}(-5+\sqrt{34})} \operatorname{sech} \frac{1}{3} \sqrt{-5+\sqrt{34}}\left[x-\frac{1}{10}(5+\sqrt{34}) t\right]
$$

with the initial condition

$$
u(x, 0)=\sqrt[4]{\frac{4}{15}(-5+\sqrt{34})} \operatorname{sech} \frac{1}{3} \sqrt{-5+\sqrt{34}} x .
$$

Firstly, we present numerical simulations in different time and space steps for $p=3$ and $p=5$, respectively, in which we take $T=10,20,30$, and 40. Some results are listed in Tables 1 and 2 for $p=3$ and $p=5$, respectively. For the simplicity of presentation, we can denote the convergence rate by $\operatorname{cor}=\frac{\|\boldsymbol{e}(\mathrm{h}, \tau)\|_{\infty}}{\|\boldsymbol{e}(\mathrm{h} / 2, \tau / 2)\|_{\infty}}$. Clearly, it verifies the second order accuracy in Theorems 3.4 and 3.5.

Table 1: The errors estimations in the sense of $L_{\infty}$ for $p=3$ at various time.

\begin{tabular}{llllll}
\hline$(\mathrm{h}, \tau)$ & & $(0.25,0.25)$ & $(0.125,0.125)$ & $(0 . .625,0.0625)$ & $(0.03125,0.03125)$ \\
\hline $\mathrm{T}=10$ & $\|e\|_{\infty}$ & $2.56674 e-3$ & $6.44399 e-4$ & $1.61280 e-4$ & $4.03336 e-5$ \\
& cor & - & 3.98315 & 3.99552 & 3.99866 \\
$\mathrm{~T}=20$ & $\|e\|_{\infty}$ & $4.48735 e-3$ & $1.12931 e-3$ & $2.82731 e-4$ & $7.07084 e-5$ \\
& cor & - & 3.97352 & 3.99430 & 3.99855 \\
$\mathrm{~T}=30$ & $\|e\|_{\infty}$ & $6.15513 e-3$ & $1.54976 e-3$ & $3.88116 e-4$ & $9.70716 e-5$ \\
& cor & - & 3.97167 & 3.99303 & 3.99825 \\
$\mathrm{~T}=40$ & $\|e\|_{\infty}$ & $7.70544 e-3$ & $1.94252 e-3$ & $4.86553 e-4$ & $1.21699 e-4$ \\
& cor & - & 3.96672 & 3.99242 & 3.99800 \\
\hline
\end{tabular}

Secondly, we simulate the wave graph of the numerical solution of the nonlinear implicit scheme (2.1)(2.3). The comparison of numerical solutions $u_{j}^{n}$ with the different time step and space step at various times is given in Figure 1 for $p=5$. The figure shows that the height of the wave graph at different time is almost identical, which implies that invariants $M$ and $E$ studied in [18] are conservative. As illustrated in Figure 1, the scheme is also stable. 
Table 2: The errors estimations in the sense of $\mathrm{L}_{\infty}$ for $\mathrm{p}=5$ at various time.

\begin{tabular}{llllll}
\hline$(\mathrm{h}, \tau)$ & & $(0.25,0.25)$ & $(0.125,0.125)$ & $(0 . .625,0.0625)$ & $(0.03125,0.03125)$ \\
\hline $\mathrm{T}=10$ & $\|e\|_{\infty}$ & $3.61271 e-3$ & $9.08877 e-4$ & $2.27537 e-4$ & $5.69039 e-5$ \\
& cor & - & 3.97492 & 3.99442 & 3.99862 \\
$\mathrm{~T}=20$ & $\|e\|_{\infty}$ & $6.67461 e-3$ & $1.68217 e-3$ & $4.21381 e-4$ & $1.05398 e-4$ \\
& cor & - & 3.96787 & 3.99203 & 3.99800 \\
$\mathrm{~T}=30$ & $\|e\|_{\infty}$ & $9.67548 e-3$ & $2.44308 e-3$ & $6.12310 e-4$ & $1.53180 e-4$ \\
& cor & - & 3.97167 & 3.99303 & 3.99825 \\
$\mathrm{~T}=40$ & $\|e\|_{\infty}$ & $7.70544 e-3$ & $1.94252 e-3$ & $4.86553 e-4$ & $1.21699 e-4$ \\
& cor & - & 3.96036 & 3.98994 & 3.99732 \\
\hline
\end{tabular}

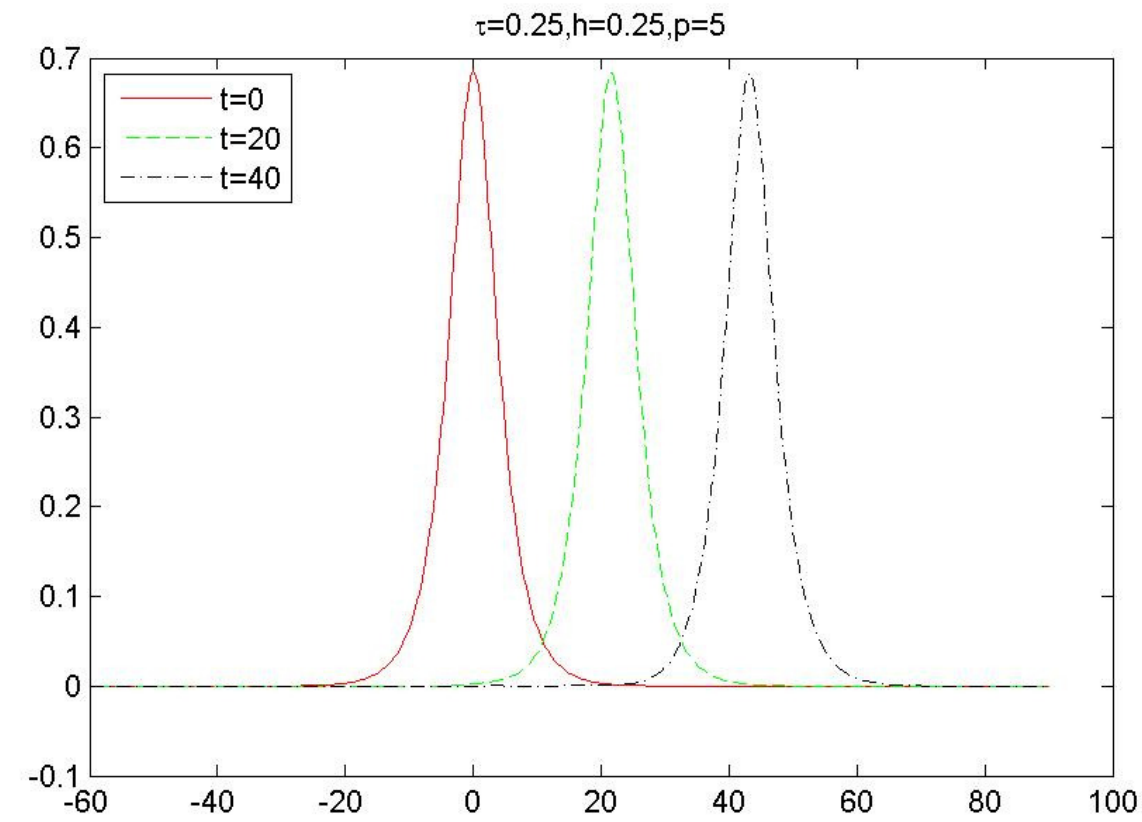

Figure 1: Wave graph of $u(x, t)$ at various time when $p=5$ and $\tau=h=0.25$.

\section{Conclusion}

In the present work, we proposed a finite difference scheme for the generalized Rosenau-KdV equation, and proved its convergence and stability. By the discrete energy method, it shows that the scheme is unconditionally stable and convergent. Numerical experiments also verify that the new scheme is reliable and efficient.

\section{Acknowledgment}

This work is supported by the Scientific Research Innovation Team Project of Yangtze Normal University (No. 2016XJTD01) and the Research Project of Chongqing Science and Technology Committee (No. CSTC2016jcyj-A0492). 


\section{References}

[1] K. Cheng, W.-Q. Feng, S. Gottlieb, C. Wang, A Fourier pseudospectral method for the "good" Boussinesq equation with second-order temporal accuracy, Numer. Methods Partial Differential Equations, 31 (2015), 202-224. 1

[2] S. K. Chung, Finite difference approximate solutions for the Rosenau equation, Appl. Anal., 69 (1998), 149-156. 1

[3] G. Ebadi, A. Mojaver, H. Triki, A. Yildirim, A. Biswas, Topological solitons and other solutions of the Rosenau-KdV equation with power law nonlinearity, Romanian J. Phys., 58 (2013), 3-14. 1

[4] A. Esfahani, Solitary wave solutions for generalized Rosenau-KdV equation, Commun. Theor. Phys. (Beijing), 55 (2011), 396-398. 1, 4

[5] J.-S. Hu, Y.-C. Xu, B. Hu, Conservative linear difference scheme for Rosenau-KdV equation, Adv. Math. Phys., 2013 (2013), 7 pages. 1

[6] J.-S. Hu, K.-L. Zheng, Two conservative difference schemes for the generalized Rosenau equation, Bound. Value Probl., 2010 (2010), 18 pages. 1

[7] Y. Luo, Y.-C. Xu, M.-F. Feng, Conservative difference scheme for generalized Rosenau-KdV equation, Adv. Math. Phys., 2014 (2014), 7 pages. 1, 3

[8] K. Omrani, F. Abidi, T. Achouri, N. Khiari, A new conservative finite difference scheme for the Rosenau equation, Appl. Math. Comput., 201 (2008), 35-43. 1

[9] P. Razborova, H. Triki, A. Biswas, Perturbation of dispersive shallow water waves, Ocean Eng., 63 (2013), 1-7. 1, 4

[10] A. Saha, Topological 1-soliton solutions for the generalized Rosenau-KdV equation, Fund. J. Math. Phys., 2 (2012), 19-25. 1,2

[11] X.-J. Yang, A new integral transform method for solving steady heat-transfer problem, Therm. Sci., 20 (2016), S639-S642. 1

[12] X.-J. Yang, A new integral transform operator for solving the heat-diffusion problem, Appl. Math. Lett., 64 (2017), 193197. 1

[13] X.-J. Yang, F. Gao, A new technology for solving diffusion and heat equations, Therm. Sci., 21 (2017), 133-140. 1

[14] X.-J. Yang, J. A. Tenreiro Machado, D. Baleanu, C. Cattani, On exact traveling-wave solutions for local fractional Korteweg-de Vries equation, Chaos, 26 (2016), 5 pages. 1

[15] K.-L. Zheng, J.-S. Hu, High-order conservative Crank-Nicolson scheme for regularized long wave equation, Adv. Difference Equ., 2013 (2013), 12 pages. 1

[16] M.-B. Zheng, J. Zhou, An average linear difference scheme for the generalized Rosenau-KdV equation, J. Appl. Math., 2014 (2014), 9 pages. 1

[17] Y. L. Zhou, Applications of discrete functional analysis to the finite difference method, International Academic Publishers, Beijing, (1991). 3

[18] J. Zhou, M.-B. Zheng, R.-X. Jiang, The conservative difference scheme for the Generalized Rosenau-KDV equation, Therm. Sci., 20 (2016), S903-S910. 1, 3, 4

[19] J.-M. Zuo, Y.-M. Zhang, T.-D. Zhang, F. Chang, A new conservative difference scheme for the general Rosenau-RLW equation, Bound. Value Probl., 2010 (2010), 13 pages. 1 